\title{
Der outlook-Kalender als zentrale Informationsplattform für die Abteilung
}

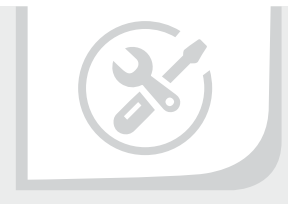

A. Wiedemann,

\begin{abstract}
Das Problem stellt sich alltäglich und vielgestaltig dar: In der Abteilungsorganisation von urologischen Krankenhausabteilungen oder Praxen müssen stationäre und ambulante Patienten, Sprechstunden, vorstationäre Termine, Urlaube, Fortbildungen, Konferenzen, und manchmal auch private Termine koordiniert und für alle sichtbar gemacht werden. Häufig existieren noch Einbestellbücher auf jeder einzelnen Station oder Ambulanz, Sprechstundenkalender, OP-Bücher... „Unfälle“ lassen dann nicht lange auf sich warten: an der Anmeldung steht ein Patient und keiner weiß, was geschehen soll, wer ihn einbestellt hat und wozu. Langwierige, nerv-raubende Rückfragen und unzufriedene Patienten sind die Folge
\end{abstract}

In unserer Abteilung hat sich ein einziger outlook-Kalender als zentrale Organisationsplattform bewährt. Diesen haben wir entsprechend unserer Anforderungen konfiguriert. Es können durch farbliche Markierungen stationäre, ambulante, vorstationäre und Sprechstunden-Patienten für verschiedene Sprechstunden
(Privat-Sprechstunde, Ermächtigungssprechstunden, Spezialsprechstunden, Angehörigensprechstunden usw.) optisch auf einen Blick sichtbar gemacht werden. Stichwortartig werden neben persönliche Daten, Diagnose, geplanter Eingriff und Besonderheiten (Zuweiser, eventuelle Immobilität, Verwandter von... usw.) ver-

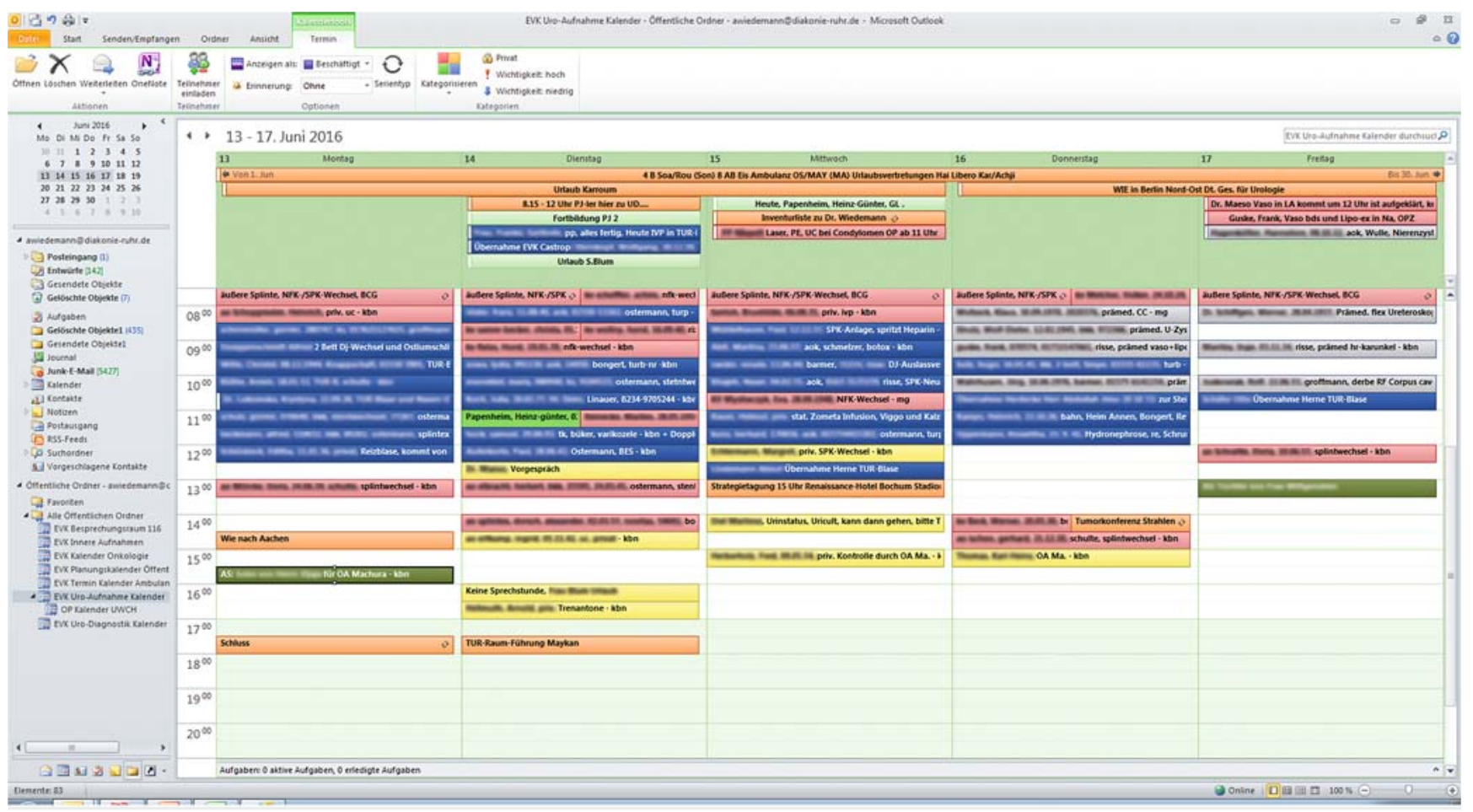

Abb. 1 Wochenansicht des Einbestellkalenders in Witten: farbliche Markierung von stationären und ambulanten, privaten und prästationären Terminen; Eintragung der Mitarbeiterverteilung („Station“, „Ambulanz“ usw.), Urlaube, Kongresse, Fortbildungen .... Alle Informationen auf einen Blick sorgen für eine reibungslose Steuerung von verschiedenen Patientenströmen. 
merkt. Auch ist es möglich, Fortbildungstermine oder Kongresse so zu terminieren, dass sie einerseits „freigehalten“ werden können, andererseits auch wahrgenommen werden können. Dauertermine, die oberhalb des eigentlichen Kalenders erscheinen, zeigen an, wer sich z. B. in Urlaub befindet oder wie die Arbeitsverteilung von Mitarbeitern (z.B. „Mitarbeiter A: Station“, „Mitarbeiter B: Ambulanz“) ist. Sogar Geburtstage oder Jubiläen können - wenn gewünscht - eingetragen werden. Bei privaten Terminen ist es möglich, deren Inhalt nicht anzeigen zu lassen. Wird ein Termin geöffnet, zeigt die Signatur an, wer den Eintrag getätigt hat - so sind eventuelle Rückfragen möglich.

Eine intelligente Rechtevergabe erlaubt es zudem, zwischen einem „Schreibrecht“, das in unserer Abteilung alle Ärzte und Sekretärinnen haben und einem „Leserecht“ für die Stationen, OP’s usw. zu unterscheiden.

Damit ist in unserer Abteilung der outlook-Kalender eine zentrale Informationsplattform geworden, der wesentlich zu einem reibungslosen organisatorischen Ablauf beigetragen hat und gegenüber einem elektronischen OP-Buch den Vorteil besitzt, dass sich nur nicht nur reine OP-Termine eingetragen finden.

\section{Interessenkonflikt}

Beratungstätigkeit: Dr. Pfleger, Pfizer; Vortragstätigkeit: Allergan, AMS Deutschland, Astellas, Berlin-Chemie, Janssen, Lilly Deutschland, Dr. Pfleger, Pfizer, Pohl-Boskamp; Studienfinanzierung: AMS Deutschland

\section{Korrespondenzadresse}

PD Dr. Andreas Wiedemann

Evangelisches Krankenhaus gGmbH,

Pferdebachstr. 27

58455 Witten

Tel.: 02302/175-2521

Fax: 02302/175-2075

E-Mail: awiedemann@diakonie-ruhr.de

Bibliografie

DOI https://doi.org/10.1055/s-0043-109974

Akt Urol 2017; 48: 279-280

(c) Georg Thieme Verlag KG Stuttgart · New York ISSN 0001-7868 